\section{In search of a cure for CJD}

\author{
Many thousands of \\ people may be \\ incubating the human \\ form of BSE. Could \\ drugs or other \\ therapeutic agents \\ prevent them from \\ succumbing? Clare \\ Thompson reports on \\ the race against time to \\ find a treatment.
}

$\mathrm{N}$ o one knows how many more people will join the 92 individuals who have so far fallen victim to the human form of bovine spongiform encephalopathy (BSE). With luck, there may never be more than a few hundred deaths from variant Creutzfeldt-Jakob disease (vCJD), as human BSE is known. But experts cannot rule out the awful possibility of an epidemic affecting many thousands of people which is why finding a way to prevent the deadly neurological damage wrought by the rogue proteins, or prions, thought to cause these diseases is a top priority.

As with efforts to establish the scale and distribution of the BSE epidemic in Europe's cattle (see pages 658-659), much depends on the ability of scientists to devise diagnostic tests that can spot infection before any symptoms appear. By the time the symptoms of vCJD have become apparent, the patient's brain is probably irretrievably damaged. "It is far too late," says Moira Bruce, a prion disease researcher at the Institute for Animal Health's Neuropathogenesis Unit in Edinburgh.

But if a reliable diagnostic test can be found, research from the labs of leading prion researchers suggests that it might just be possible to save infected people from the nightmare of vCJD. Whether therapies can be developed on the timescale required, however, remains to be seen. And even if they can, public subsidies will probably be needed to bring them to market.

The first signs that drugs might delay the onset of prion diseases came in the mid1980s, from work on scrapie, a prion disease of sheep. In those days, BSE was unheard of,

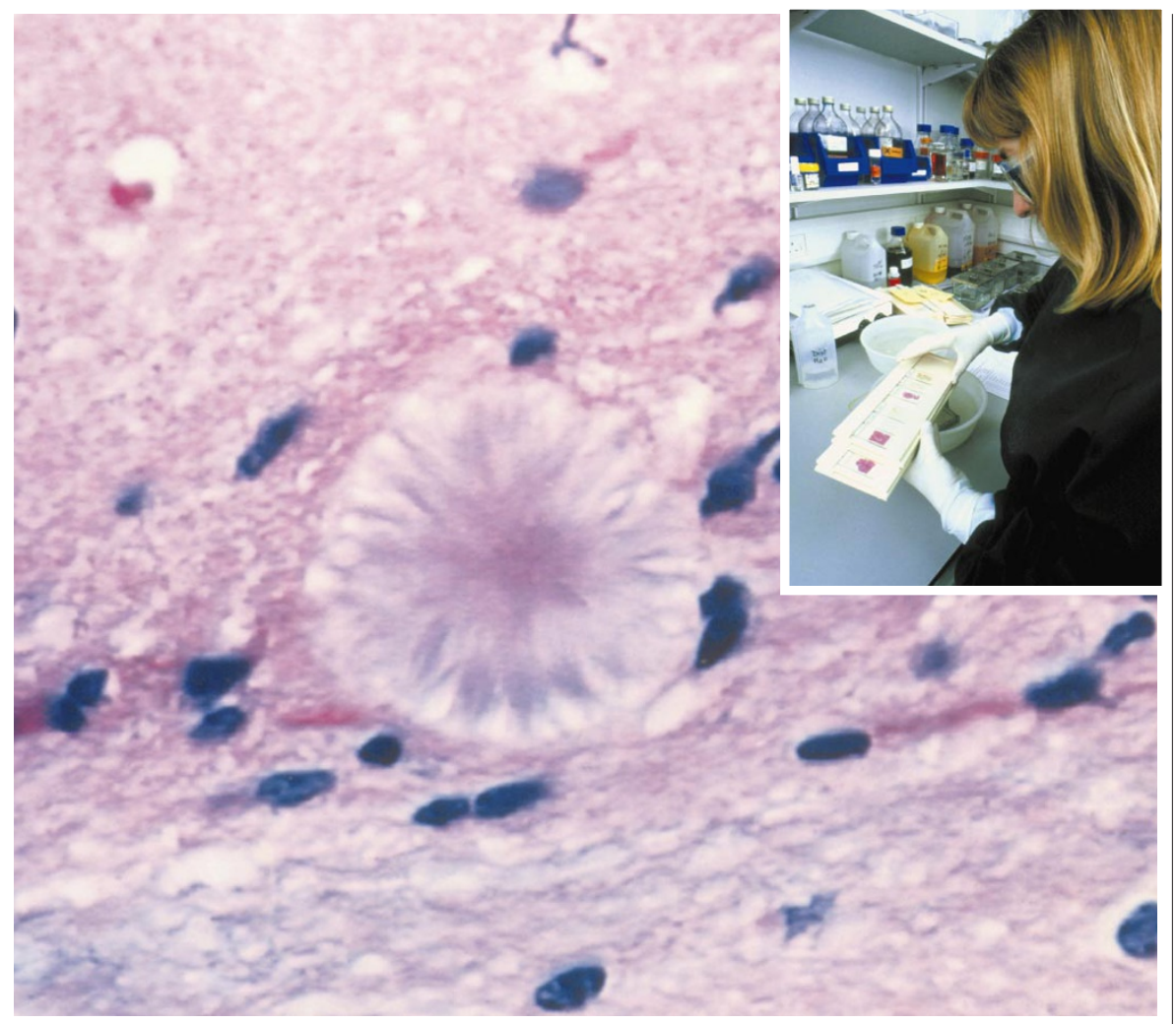

and CJD was a neurological curiosity - the sporadic form of the disease, unrelated to BSE, arises in about one in a million people. Indeed, the veryidea that these diseases might be caused by a misshapen protein converting a normal brain protein, called PrP, into its own, twisted form was then outside the scientific mainstream - most researchers believed that a virus was involved.

\section{Slow-motion scrapie}

In 1984, Heino Diringer and Bernhard Ehlers of the Robert Koch Institute in Berlin showed that an antiviral drug called dextran sulphate could prolong scrapie's incubation period in mice infected with the disease ${ }^{1}$. Dextran sulphate belongs to a class of compounds known as polyanions, and other members of this group seem to have stronger effects - Bruce and her colleagues in Edinburgh, for instance, are working with a less toxic compound called pentosan polysulphate, normally used to treat cystitis ${ }^{2}$.

Following Diringer and Ehlers's original discovery, a few other compounds have been found that also delay the onset of scrapie symptoms in rodent models of the disease. In 1989, researchers led by Maurizio Pocchiari of the Italian National Institute of Health in Rome showed that an antifungal drug called amphotericin B pulls off the same trick as dextran sulphate ${ }^{3}$ - and again, prion researchers are now working with more effective derivatives ${ }^{4}$.

Since then, the list of candidate drugs has continued to grow ${ }^{5-9}$. And with drug companies starting to screen their huge libraries of
Irreversible damage? The characteristic plaques formed in brain tissue as a result of vCJD cause neurodegeneration and death. should be on the way. John Collinge of Imperial College, London, for instance, is now collaborating with GlaxoSmithKline. "We are working on a high-throughput approach, looking at about 200,000 compounds to see which ones can stabilize PrP," he says.

\section{Into the unknown}

The problem is that for most of the candidate drugs, there is no clear idea of why they delay the onset of symptoms. Many of them are presumed to work by interfering with the conversion of normal PrP to the rogue, prion form, known as $\operatorname{PrP}^{\mathrm{Sc}}$. But without a detailed understanding of how the compounds work, it is difficult to design

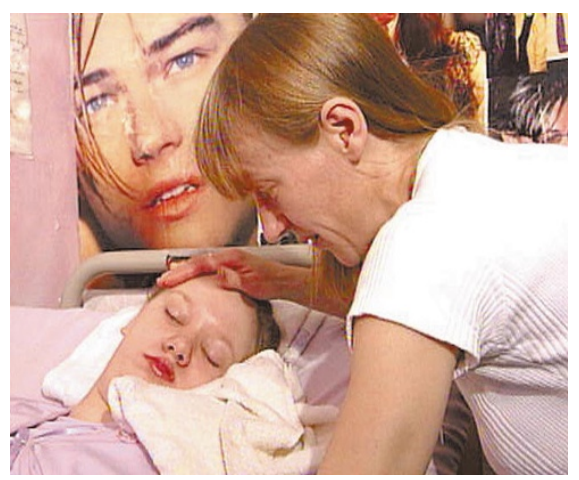

Prion victim: Zoe Jeffries is the youngest person so far to succumb to variant CJD. She died last October at the age of 14 . compounds for potential CJD drugs, more 


\section{$W_{\text {numana }}^{\text {ened }}$} Government may have to work hand in hand

\section{with a pharmaceutical company.}

improved derivatives that could be developed into effective drugs.

This is why some experts believe that a more targeted approach may be necessary. One of the most exciting recent discoveries in this area comes from the group led by Claudio Soto of the Serono Pharmaceutical Research Institute in Geneva. Prions differ from normal PrP in that parts of the protein have been converted from one structural conformation, known as an $\alpha$-helix, to another, called a $\beta$-sheet. "Several years ago, evidence surfaced that if a protein doesn't have a proline amino acid, then it is more likely to adopt a $\beta$-sheet formation," says Soto. "This prompted us to use proline to keep the structure of the $\alpha$-helix intact."

Soto and his colleagues devised a sequence of 13 amino acids, called iPrP13, that contains three prolines, but otherwise

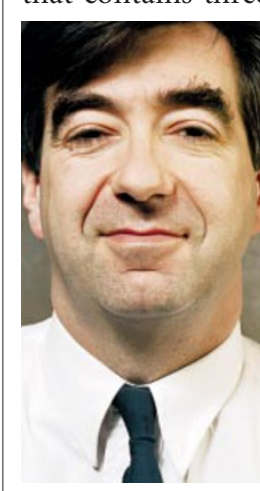

John Collinge: hopes antibodies may defeat prions. convert $\operatorname{PrP}$ into $\operatorname{PrP}{ }^{S c}$. They have identified a region within $\mathrm{PrP}$ that appears to interact with protein X (ref. 11). Focusing on compounds with structures that can bind to this region, the San Francisco team has screened for those that inhibit the conversion of PrP to $\mathrm{PrP}^{\mathrm{Sc}}$ - eventually finding two that did the job and also had low toxicity ${ }^{12}$. But Cohen admits that they are some way from having a viable drug. "The activity would have had to be 100-fold more potent," he says.

\section{Prion patrol}

Other researchers wonder whether it might be possible to prevent the deadly prions from getting into the brain in the first place. Before they enter the central nervous system, prions multiply in immune cells known as follicular dendritic cells. These cells can be temporarily inactivated using a soluble form of a protein called lymphotoxin- $\beta$ receptor. And last year, two teams, one led by Bruce, the other by Charles Weissmann of Imperial College and Adriano Aguzzi of the University of Zurich, showed that this treatment could delay the onset of scrapie in mice ${ }^{13,14}$.

Perhaps the most intriguing possibility has been borrowed from Alzheimer's disease research, where a vaccine to generate antibodies against a protein called amyloid- $\beta$ is showing good promise as a treatment ${ }^{15,16}$. Collinge and Cohen are among the researchers now experimenting with antibodies against $\operatorname{PrP}^{S c}$, to see if these can work a similar magic.

But whichever strategy proves most effective, one important obstacle will remain — money. Preventing thousands of cases of vCJD is a noble goal, but the harsh realities the drug industry demand a bigger market, if companies are to meet the huge costs of developing a therapeutic agent. "We need more evaluation and human trials," says Collinge. "And who would pay for that remains an important question. It may get to the stage where government would have to work hand in

Degenerating: an MRI scan of a human brain with vCJD.

$\approx \odot 2001$ Macmillan Magazines Ltd hand with a pharmaceutical company."

Clare Thompson is a medical writer based in London.

1. Ehlers, B. \& Diringer, H. J. Gen. Virol. 65, 1325-1330 (1984).

2. Farquhar, C., Dickinson, A. \& Bruce, M. Lancet 353, 117 (1999).

3. Pocchiari, M., Casaccia, P. \& Ladogana, A. J. Infect. Dis. 150, 795-802 (1989).

4. Adjou, K. T. et al. Antimicrob. Agents Chemother. 39, 2810-2812 (1995)

5. Caughey, B. \& Race, R. E. J. Neurochem. 59, $768-777$ (1992).

6. Tagliavini, F. et al. Science 276, 1119-1125 (1997).

7. Caughey, W. S., Raymond, L. D., Horiuchi, M. \& Caughey, B. Proc. Natl Acad. Sci. USA 95, 12117-12122 (1998).

8. Katsumi, D., Iwaki, T. \& Caughey, B. J. Virol. 74, 4894-4897 (2000).

9. Supattapone, S., Nguyen, H.-O. B., Cohen, F. E., Prusiner, S. B. \& Scott, M. R. Proc. Natl Acad. Sci. USA 96, 14529-14534 (1999).

10. Soto, C. et al. Lancet 355, 192-197 (2000).

11. Kaneko, K. et al. Proc. Natl Acad. Sci. USA 94, 10069-10074 (1997).

12. Perrier, V. et al. Proc. Natl Acad. Sci. USA 97, 6073-6078 (2000).

13. Mabbott, N. A., Mackay, F., Minns, F. \& Bruce, M. A. Nature Med. 6, 719-720 (2000).

14. Montrasio, F. et al. Science 288, 1257-1259 (2000).

15. Morgan, D. et al. Nature 408, 982-985 (2000).

16. Janus, C. et al. Nature 408, 979-982 (2000). 